\title{
Cosmic rays linked to rapid mid-latitude cloud changes
}

\author{
B. A. Laken ${ }^{1,2}$, D. R. Kniveton ${ }^{1}$, and M. R. Frogley ${ }^{1}$ \\ ${ }^{1}$ Department of Geography, University of Sussex, Falmer, Brighton, England, BN1 9QJ, UK \\ ${ }^{2}$ Instituto de Astrofísica de Canarias, 38205 La Laguna, Tenerife, Spain
}

Received: 7 June 2010 - Published in Atmos. Chem. Phys. Discuss.: 2 August 2010

Revised: 16 November 2010 - Accepted: 18 November 2010 - Published: 24 November 2010

\begin{abstract}
The effect of the Galactic Cosmic Ray (GCR) flux on Earth's climate is highly uncertain. Using a novel sampling approach based around observing periods of significant cloud changes, a statistically robust relationship is identified between short-term GCR flux changes and the most rapid mid-latitude $\left(60^{\circ}-30^{\circ} \mathrm{N} / \mathrm{S}\right)$ cloud decreases operating over daily timescales; this signal is verified in surface level air temperature (SLAT) reanalysis data. A General Circulation Model (GCM) experiment is used to test the causal relationship of the observed cloud changes to the detected SLAT anomalies. Results indicate that the anomalous cloud changes were responsible for producing the observed SLAT changes, implying that if there is a causal relationship between significant decreases in the rate of GCR flux ( $~ 0.79 G U$, where $G U$ denotes a change of $1 \%$ of the 11 year solar cycle amplitude in four days) and decreases in cloud cover $(\sim 1.9 \mathrm{CU}$, where $C U$ denotes a change of $1 \%$ cloud cover in four days), an increase in SLAT $(\sim 0.05 K U$, where $K U$ denotes a temperature change of $1 \mathrm{~K}$ in four days) can be expected. The influence of GCRs is clearly distinguishable from changes in solar irradiance and the interplanetary magnetic field. However, the results of the GCM experiment are found to be somewhat limited by the ability of the model to successfully reproduce observed cloud cover. These results provide perhaps the most compelling evidence presented thus far of a GCR-climate relationship. From this analysis we conclude that a GCR-climate relationship is governed by both short-term GCR changes and internal atmospheric precursor conditions.
\end{abstract}

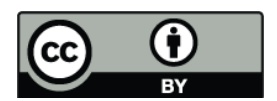

Correspondence to: B. A. Laken (blaken@iac.es)

\section{Introduction}

Evidence of links between small changes in solar activity and Earth's climate has been identified by several palaeoclimatic studies (e.g., Bond et al., 2001; Neff et al., 2001; Mauas et al., 2008). However, as yet, there is no accepted physical process which can fully account for such observations. Several theories have been suggested, and, of these, perhaps the most contentious proposes a relationship between the Galactic Cosmic Ray (GCR) flux and cloud cover (Svensmark and Friis-Christensen, 1997).

Previous studies attempting to test the validity of a GCR - cloud connection have tried to use the GCR flux to infer cloud changes; the majority of these studies have either revolved around the daily timescale, high magnitude, infrequent GCR decreases known as Forbush Decrease (FD) events (Laken et al., 2010; Laken and Kniveton, 2010), or long-term (annual-to-decadal) correlations between the GCR flux and cloud cover (Marsh and Svensmark, 2000). However, both approaches may be inherently flawed, as they assume a first-order relationship (i.e. presuming that cloud changes consistently accompany GCR changes), when instead, a second-order relationship may be more likely (i.e. that cloud changes only occur with GCR changes if atmospheric conditions are suitable). Indeed, evidence of secondorder relationships between GCR and cloud variations has been implied by the results of Harrison and Ambaum (2009). To address this issue, we adopt a fresh approach by performing a composite (epoch-superpositional) analysis based on periods of significant cloud change and then examining corresponding solar-related variations. If, under certain conditions, GCR-related effects influence cloud changes, then constructing the composite in this manner will automatically account for any dependence of a GCR - cloud connection on initial precursor conditions (as such conditions will have been met for GCR-related cloud changes to occur).

Published by Copernicus Publications on behalf of the European Geosciences Union. 
The composite sample is based around the most rapid ( $>0.95$ percentile) daily timescale decreases in short-term cloud changes occurring over mid-latitude regions. We chose to focus on this region for several reasons: (i) a prevalence of stratified clouds (which may be an important precursor condition) (Nicoll and Harrison, 2010); (ii) reliable satellite cloud retrievals compared to high- latitude regions; and (iii) past studies suggest sensitivity of cloud modulation by GCRs over such regions (Harrison and Ambaum, 2009; Tinsley and Dean, 1991; Veretenenko et al., 2007; Harrison and Stephenson, 2006). Critically, normalised units are used over absolute values, as we propose that neutral and electricallyenhanced cloud variability may be distinguished by the rapidity of their changes; this hypothesis is based on the results of model and observational studies which suggest that GCRs may (either directly or indirectly) increase the efficiency of cloud forming processes (e.g., Duplissy et al., 2010; Tinsley and Yu, 2004; Tinsley et al., 2006; Yu and Turco, 2008). Short-term cloud change are calculated from the infrared (IR) retrieved International Satellite Cloud Climatology Project (ISCCP) D1 dataset, while GCR values are based on an amalgamated dataset of neutron monitors from locations across the globe. ISCCP data is an inter-calibration of data recorded from a fleet of international satellites. It provides long-term, global cloud coverage, for a wide range of cloud and atmospheric variables (Rossow et al., 1996). In this work we have used total cloud amount (VIS/IR), and IR cloud amount at seven different pressure levels at intervals between 30$1000 \mathrm{mb}$. ISCCP data is provided on an equal-area grid of $280 \times 280 \mathrm{~km}^{2}$. Chief limitations associated with the data include issues of artificial cloud changes resulting from satellite drifts and inter-calibration problems, and also viewing limitations (i.e. viewing of low clouds may be obscured by presence of higher clouds directly above). These, and additional problems, are considered in detail by several research groups (e.g., Evan et al. 2007; Campbell, 2004; Pallé, 2005, and references therein).

\section{Data and methods}

The epoch-superposition (composite) methodology used in this study is similar to the approach of previous FD based studies (see Laken and Kniveton, 2010; Todd and Kniveton, 2001). The composite is constructed to represent the largest (top 5\%) decreases in short-term cloud changes over the midlatitude $\left(60^{\circ}-30^{\circ} \mathrm{N} / \mathrm{S}\right)$ regions (i.e. the most rapid decreases in cloud cover over the mid-latitudes) detected by the IR retrieved ISCCP D1 (Rossow et al., 1996) dataset between 1986 to 2006 . The dates of the top $5 \%$ cloud changes are selected by firstly calculating the differential cloud change over area-averaged mid-latitude regions, (where the differential cloud change $(d c c)$ of each day is equal to daily average cloud change $(x)$, minus an averaging period of three days which begins five days prior to each date, as illustrated in
Eq. (1). Each $d c c$ value is then ranked, and then the dates with the largest negative changes are compiled. This composite is then filtered to remove dates which were coincident within a -10 to +3 day period of preceding dates to remove consecutive dates from the composite.

$d c c^{n}=x^{n}-\frac{\sum\left(x^{n-5}+x^{n-4}+x^{n-3}\right)}{3}$

In this study, normalised units are used instead of absolute values; these are a first order derivative, determined by taking the difference of an average daily value against a moving three day averaging period beginning five days prior to each date (identical to Eq. 1). The two-day interval between the averaging period and the differenced date accounts for temporal autocorrelation present in the data. GCR data for this study are drawn from nine different barometric pressure-adjusted neutron monitor datasets, selected for both their long term monitoring records and their global distribution (these are namely: Apatity, Climax, Kiel, Magadan, Mcmurdo, Moscow, Newark, South Pole and Thule). Shortterm GCR changes are calculated for each individual neutron monitor; the values are then all calibrated to be relative to the peak-to-peak GCR flux changes experienced at each individual neutron monitor over the 11-year solar cycle and finally integrated into one dataset. Thus, the units of GCR changes used here are given as " $G U$ ", defined as a change of $1 \%$ of the 11-year solar cycle amplitude in four days. All other units given in this work are similarly defined, where temperature change is denoted " $K U$ " (a change of $1 \mathrm{~K}$ in four days) and cloud change is denoted " $C U$ " (a change of $1 \%$ cloud cover in four days) etc.). Statistical significance of the datasets are evaluated using paired two-tailed Students T-tests, with critical T-values (at the 0.95 level) established by Monte Carlo simulations.

\section{Results}

The composite sample shows a positive correlation between statistically significant cloud changes and variations in the short-term GCR flux (Fig. 1): increases in the GCR flux occur around day -5 of the composite, and correspond to significant localised mid-latitude increases in cloud change. After this time, the GCR flux undergoes a statistically significant decrease $(\sim 1.2 G U)$ centred on the key date of the composite; these changes correspond to widespread statistically significant decreases in cloud change $(\sim 3.5 \mathrm{CU}$, $\sim 1.9 \mathrm{CU}$ globallyaveraged) over mid-latitude regions. A latitude/height profile of the key date cloud changes reveals that the anomalous cloud decreases are predominantly located at mid- to low tropospheric levels (Fig. 2). It is important to note that these anomalous changes bear a remarkable latitudinal symmetry and appear linked to the high-pressure returnflow regions associated with the Hadley cells. Comparable anomalies are identified over both IR and VIS ISCCP channels (Fig. 3). The VIS anomalies are slightly smaller than 

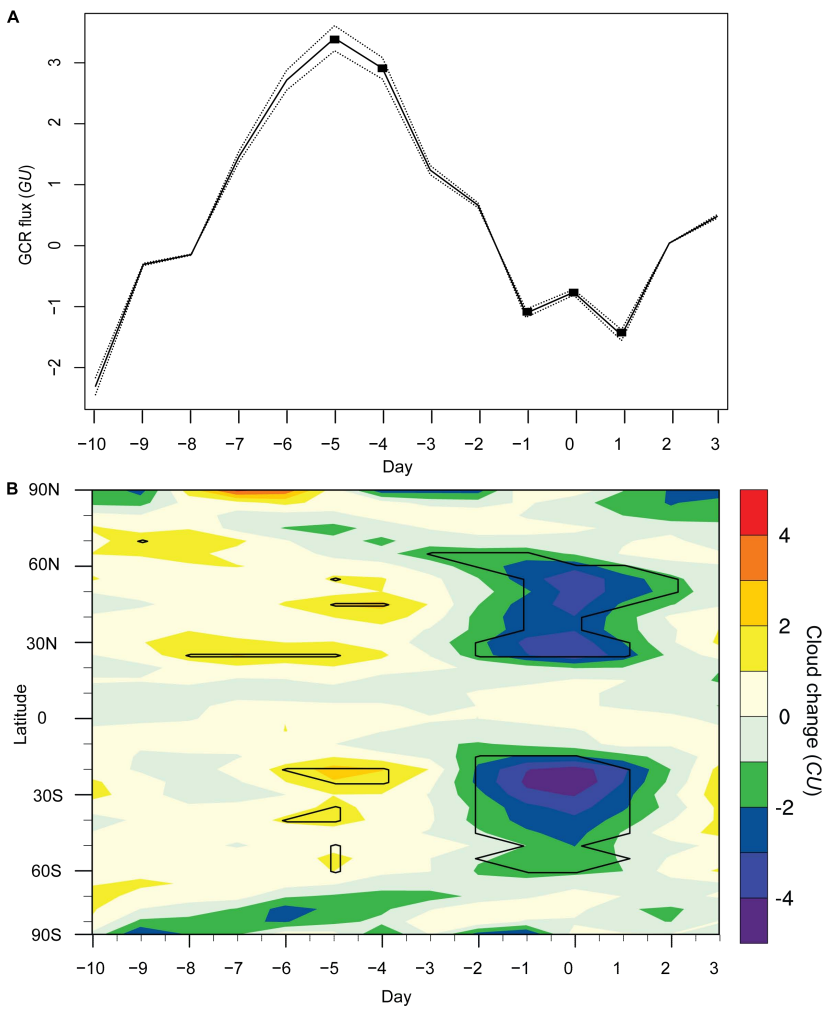

Fig. 1. (A) Short term GCR change (significance indicated by markers) and (B) anomalous cloud cover changes (significance indicated by solid contours) occurring over the composite period. GCR data sourced from multiple neutron monitors, variations normalised against changes experienced over a Schwabe cycle. Cloud changes are a tropospheric $(30-1000 \mathrm{mb})$ average from the ISCCP D1 IR cloud values.

their IR-detected counterparts; such differences between the IR/VIS channels are likely attributable to the limited observing periodicity of VIS detections, which are restricted to the circle of illumination, and are therefore unable to consider cloud amount during night-time periods. Consequently, this produces differences in the daily averaged cloud amounts observed between IR/VIS channels.

An analysis of solar interplanetary magnetic field (IMF) (Fig. 4a, b), total solar irradiance (TSI) taken from the Active Cavity Radiometer Irradiance Monitor (ACRIM) reconstructions (Willson and Mordvinov, 2003) (Fig. 4c), and ultraviolet (UV) activity (Fig. 4d) suggests that the IMF and irradiance parameters demonstrate some (non-significant) variability over the composite period; when considered together, these changes likely indicate the occurrence of short-term minima/maxima in solar activity that are possibly related to solar Carrington rotations. However, only the rate of GCR flux undergoes correlated and statistically significant co-temporal variations to cloud changes over the composite period. These results suggest that the effects we observe are independent of other solar phenomena and that we can

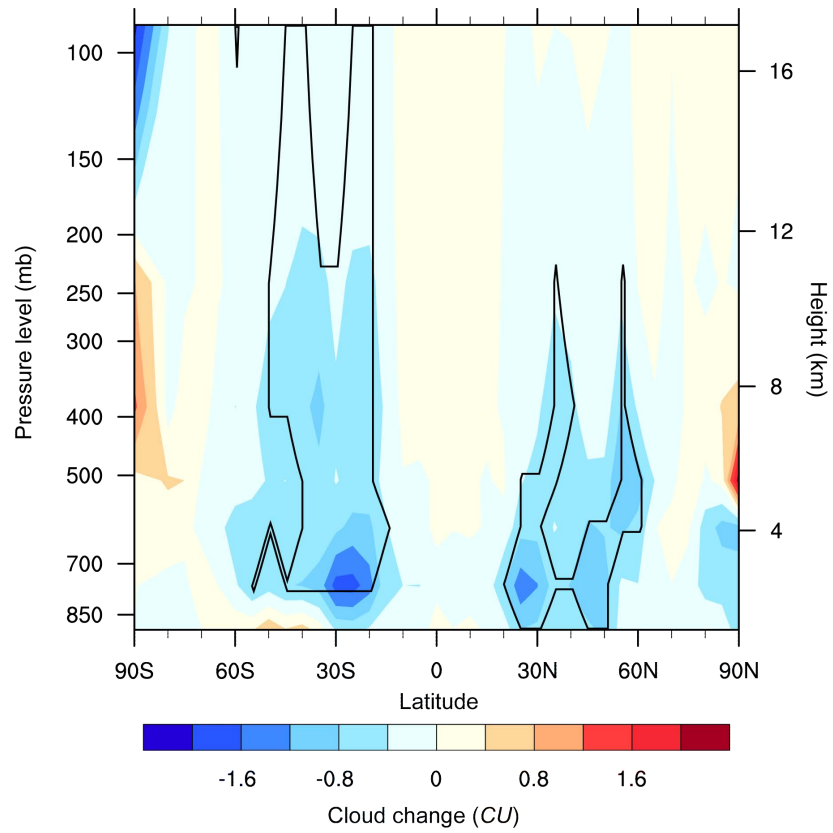

Fig. 2. A latitude/height profile of anomalous cloud cover changes occurring on the key date of the composite. Statistically significant changes (above the 0.95 confidence level) are indicated by solid contours.

therefore discount the possibility that an alternative solarterrestrial mechanism is operating (such as those detailed by: Tinsley, 2008; Douglas and Clader, 2002; Haigh, 1996; Kniveton et al., 2003).

During the key day mid-latitude cloud cover decreases, NCEP/NCAR reanalysis data (Kalany et al., 1996) show that the surface level air temperature (SLAT) underwent a statistically significant increase of $\sim 0.1 K U$ over midlatitude regions $(\sim 0.05 \mathrm{KU}$ as a global average) (Fig. 5a); this temperature forcing is consistent with the radiative impacts of decreasing mid- to low-level cloud cover over midlatitudes. Although a correlation between cloud changes and the GCR flux is evident, it cannot be determined if the cloud/SLAT changes are causally related to the GCR, or if the cloud/SLAT anomalies are merely a product of internal climate variations. To address this issue, a HadAm3 (van der Wal, 1998) General Circulation Model (GCM) experiment is conducted which attempts to reproduce the observed midlatitude anomalous cloud changes; this is done by directly manipulating the SW/LW radiation schemes through the use of code-altering inserts (known as modscripts), which artificially increase/decrease cloud amount over prescribed areas and times, by the use of scale factors based on the observed anomalous cloud changes. The series of forced cloud changes were repeated in regularly-spaced temporal intervals, over several 5-year runs, these were then composited, thereby constructing a type of initial-condition ensemble, equivalent to the temporal period of the observations (in 

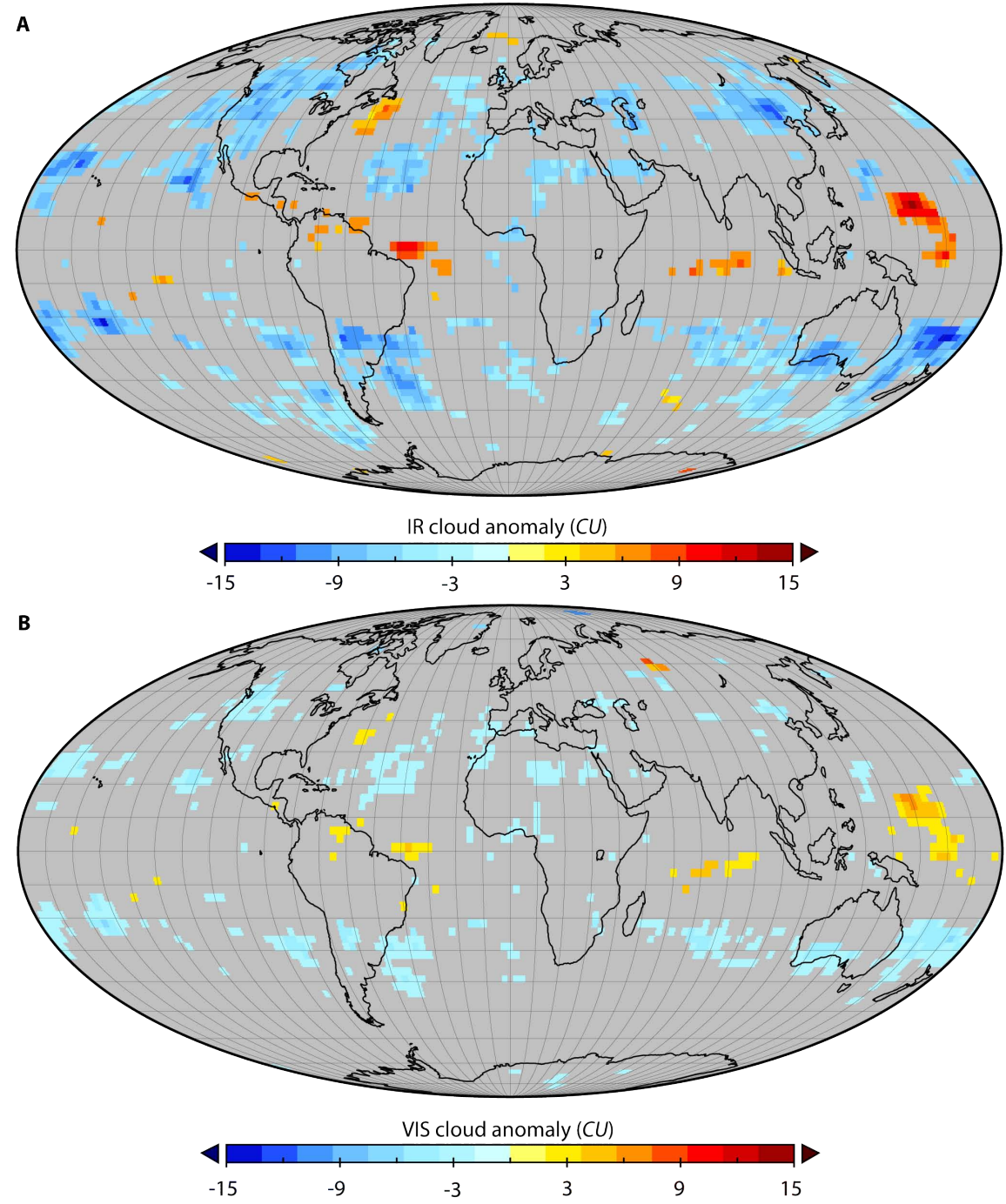

Fig. 3. Anomalous rates of cloud changes $(30-1000 \mathrm{mb})$ displayed over the globe for both (A) IR and (B) VIS ISCCP D1 channels; only locally significant ( 0.95 confidence level) anomalies are displayed.

both number of events and the composite period). The HadAm3 model was run at the standard N48 resolution, with 19 vertical levels.

The GCM results successfully produced a pattern of SLAT increase comparable to observations (Fig. 5b), implying a causal relationship between the observed cloud-SLAT changes. Between days -5 to +3 (after the first appearance of significant cloud changes) the observed correlation coefficient between SLAT and cloud cover was found to be $R=-0.91$. Over the same period, the GCM demonstrated a correlation coefficient of $R=-0.93$, indicating that the observed cloud-climate relationship was reproduced by the GCM. However, the magnitude of the SLAT changes was far smaller than that observed (by $\sim 75 \%$ ) and the anomalies did not demonstrate any statistical significance. These differences may be attributed to the shortcomings of HadAm3 cloud scheme, which has been shown to largely underestimate cloud cover over the globe (Webb et al., 2001; Zhang et al., 2005). Underestimation of cloud amount has the effect of restricting the cloud amount which can be altered during the GCM experiment (i.e. cloud can only be reduced if it pre-exists within the GCM, as the model which underproduces cloud this may present a limiting factor upon the experiment).

\section{Discussion}

The strong and statistically robust connection identified here between the most rapid cloud decreases over mid-latitude regions and short-term changes in the GCR flux is clearly distinguishable from the effects of solar irradiance and IMF variations. The observed anomalous changes show a strong 


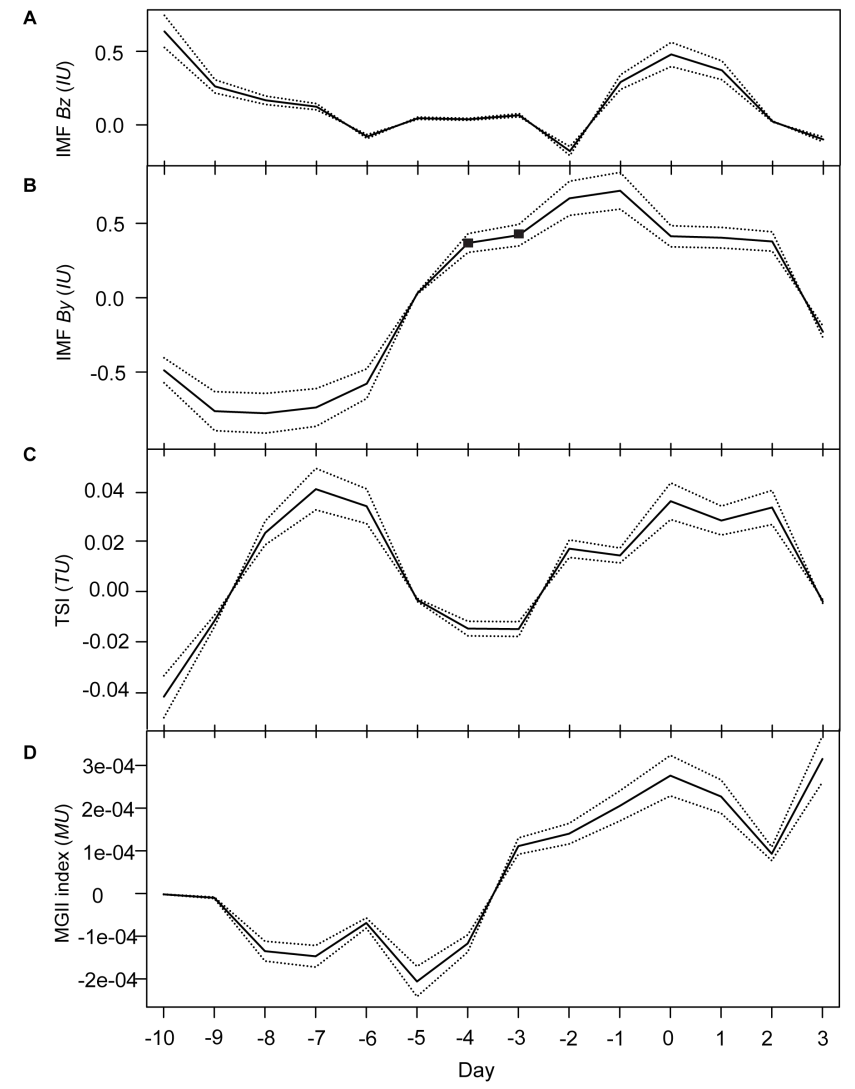

Fig. 4. Anomalous changes in the (A) IMF $B z$ (north-south) component, (B) IMF By (east-west) component, (IMF units in "IU", denoting a change of $1 \mathrm{n} / \mathrm{T}$ in four days] (C) Total Solar Irradiance (TSI) from the Active Cavity Radiometer Irradiance Monitor (ACRIM) reconstructions (units in " $T U$ ", denoting a change of $1 \mathrm{~W} / \mathrm{m}^{2}$ in four days) and (D) MG II UV index (units in " $M U$ ", denoting a change of 1 unit in four days). Statistically significant anomalies indicated by markers, dotted line indicates 0.95 confidence level.

latitudinal symmetry around the equator; alone, this pattern gives a good indication of an external forcing agent, as there is no known mode of internal climate variability at the timescale of analysis, which could account for this distinctive response. It is also important to note that these anomalous changes are detected over regions where the quality of satellite-based cloud retrievals is relatively robust; results of past studies concerned with high-latitude anomalous cloud changes have been subject to scrutiny due to a low confidence in polar cloud retrievals (Laken and Kniveton, 2010; Todd and Kniveton, 2001) but the same limitations do not apply here.

Although mid-latitude cloud detections are more robust than those over high latitudes, Sun and Bradley (2002) identified a distinctive pattern of high significance between GCRs and the ISCCP dataset over the Atlantic Ocean that corresponded to the METEOSAT footprint. This bias does not appear to influence the results presented in this work: Fig. 6 shows the rates of anomalous IR-detected cloud change occurring over Atlantic, Pacific and land regions of the midlatitudes during the composite period, and a comparable pattern of cloud change is observed over all regions, indicating no significant bias is present.

Additional considerations should be given to the results of Kristjánsson et al. (2002), who demonstrated the presence of large differences between IR and VIS ISCCP cloud retrievals; a low confidence should be placed in detected cloud changes where IR and VIS cloud detections disagree. However, the results presented in this work identify comparable, locally significant anomalous cloud changes over both IR and VIS channels (Fig. 3), confirming that the IR-detected anomalies are reliable.

There are two theoretical micro-physical mechanisms via which a GCR-cloud link may function: the first, referred to as ion-mediated nucleation (IMN), operates by the catalysation of new ultrafine condensation nuclei (of $<2 \mathrm{~nm}$ in diameter) from trace condensable atmospheric vapours by ions (Raes et al., 1986). Through condensation and coagulation these nuclei may grow to sizes of $\sim 100 \mathrm{~nm}$ in diameter, becoming sufficiently large enough to act as cloud condensation nuclei (CCN) (Yu et al., 2008). CCN concentration controls cloud droplet number concentration, which influences macro-scale cloud properties such as reflectivity as well as rainfall generation efficiency in low level stratus clouds. As rainfall processes are an important factor influencing cloud lifetimes, changes in rainfall generation may potentially alter the average cloudiness of a region (Carslaw et al., 2002).

The second mechanism is known as the near-cloud effect, and functions via a link between the global electric circuit (GEC) and cloud microphysics. The GEC is made up of the ionosphere (which is maintained at a potential of $\sim 250 \mathrm{kV}$ by upward current from thunderstorms and other electrified clouds) and the vertical current density, which flows downwards from the ionosphere to the Earth's surface (at $\sim 1$ $6 \mathrm{pAm}^{-2}$ ) over all fair-weather regions. The GCR flux maintains the atmosphere as a weakly conducting plasma, as a result variations in the GCR flux modulate the vertical current density. Since clouds effectively scavenge small ions, electrical conductivity within clouds is far lower than in surrounding clean air regions. Consequently, cloud boundaries act as conductivity gradients, resulting in charge accumulation. As a result, charges of $\sim 100 \mathrm{e}$ are transferred to aerosols and cloud droplets at cloud boundaries (Nicoll and Harrison, 2010), which may affect aerosol scavenging rates (Tinsley et al., 2001; Tripathi and Harrison, 2002). It has been suggested that this effect may be significant to the concentrations of both CCN and ice forming nuclei (IFN) within clouds, and as a result may influence macro-scale cloud properties. However, the exact effect resulting from such a mechanism is unclear, as scavenging rates appear to be a complex function of droplet/particle size, concentration, and charge density (Tinsley et al., 2007). 

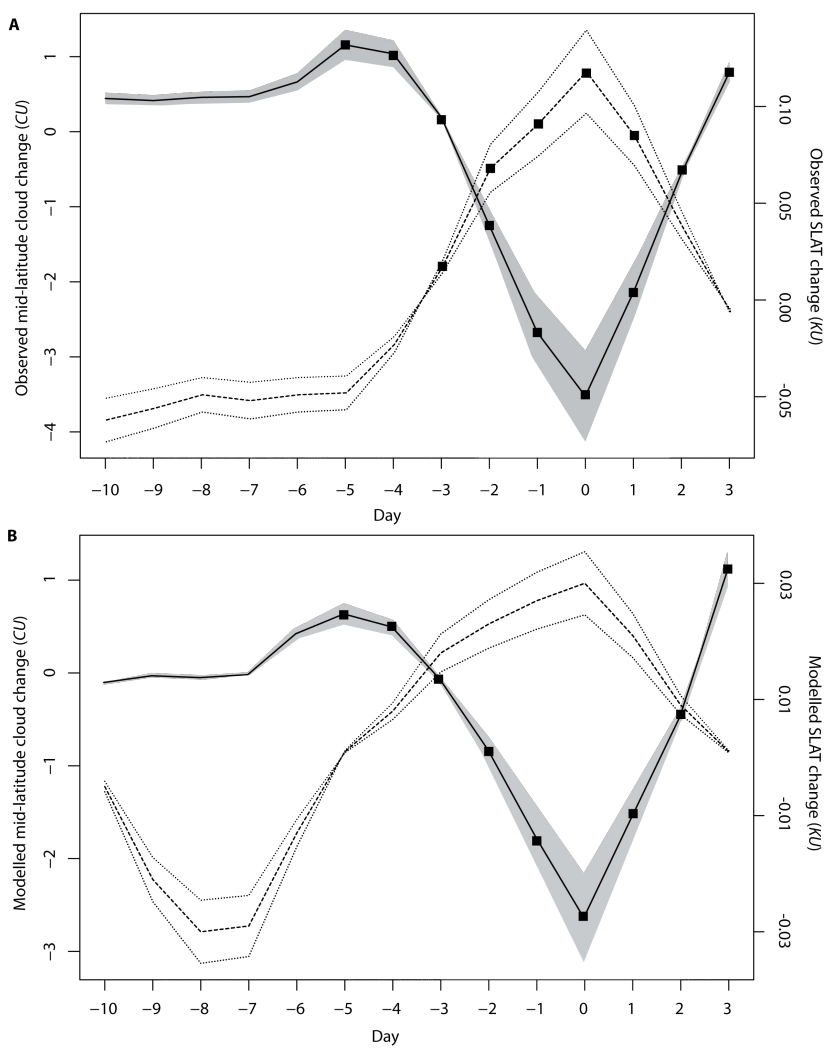

Fig. 5. Anomalous mid-latitude $\left(60^{\circ}-30^{\circ} \mathrm{N} / \mathrm{S}\right)$ average tropospheric (30-1000 mb) cloud cover changes (solid line) and surface level air temperature changes (dashed line) from (A) observations and (B) the HadAM3 GCM. Markers indicate statistically significant anomalies (above the 0.95 confidence level). Solid grey shade and dotted lines indicate the 0.95 level confidence intervals for mean cloud and air temperature changes, respectively.

Although the results presented here are not suitable to provide detail regarding the potential microphysical processes related to a GCR-cloud link, we may speculate based on our observations: aerosol impoverished clean-air oceanic regions are thought to be the most likely locations for an IMN mechanisms to be of consequence to cloud cover (Rosenfeld et al., 2006; Kristjánsson et al., 2008; Yu et al., 2008). The location of the observed anomalous cloud changes, over mid-to-low tropospheric levels occur over both land and ocean regions (Fig. 6). Cloud changes over land are found to be larger than those over ocean regions; this implies that these changes are not dependent on regional aerosol properties (which are distinct between land and ocean regions). Therefore, if a GCR related process is responsible for influencing the observed cloud anomalies this suggests it may be linked to the GEC rather than to an IMN process.

It should be noted that although the GCM experiment corroborates the observed link between changes in cloudiness and SLAT, it does not provide any further information on the

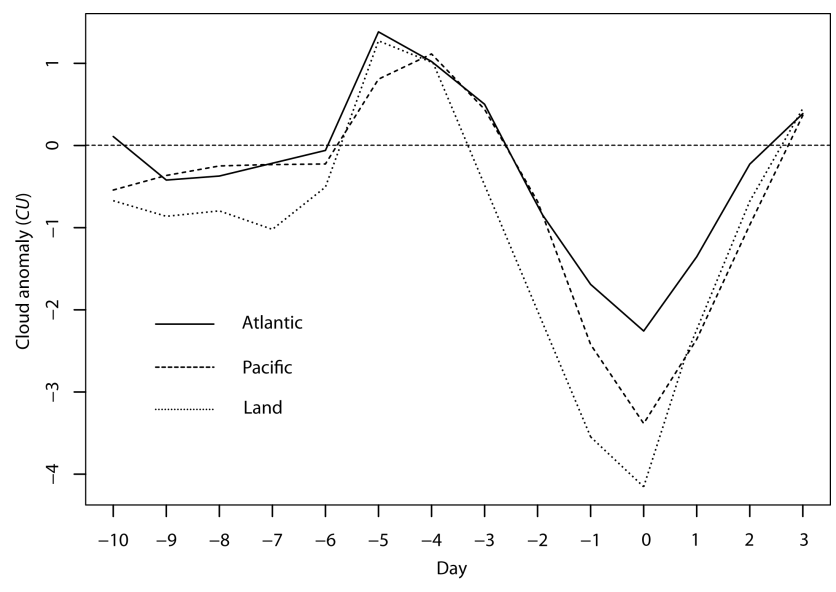

Fig. 6. The mean rate of IR-detected cloud change $(30-1000 \mathrm{mb})$ over mid-latitude regions between days -10 and +3 , over land regions (dotted line) and Pacific/Atlantic ocean areas (solid and dashed lines respectively).

suggested link between GCR and cloud cover. Based on the relationships observed in this study, and assuming that there is no linear trend in the short-term GCR change, we speculate that little $\left(\sim 0.088^{\circ} \mathrm{C} /\right.$ decade $)$ systematic change in temperature at mid-latitudes has occurred over the last 50 years. However, at shorter time-scales this phenomenon may contribute to natural variability, potentially reducing detectability of an anthropogenic signal.

\section{Conclusions}

This work has demonstrated the presence of a small but statistically significant influence of GCRs on Earth's atmosphere over mid-latitude regions. This effect is present in both ISCCP satellite data and NCEP/NCAR reanalysis data for at least the last 20 years suggesting that small fluctuations in solar activity may be linked to changes in the Earth's atmosphere via a relationship between the GCR flux and cloud cover; such a connection may amplify small changes in solar activity. In addition, a GCR - cloud relationship may also act in conjunction with other likely solar - terrestrial relationships concerning variations in solar UV (Haigh, 1996) and total solar irradiance (Meehl et al., 2009). The climatic forcings resulting from such solar - terrestrial links may have had a significant impact on climate prior to the onset of anthropogenic warming, accounting for the presence of solar cycle relationships detectable in palaeoclimatic records (e.g., Bond et al., 2001; Neff et al., 2001; Mauas et al., 2008).

Further detailed investigation is required to better understand GCR - atmosphere relationships. Specifically, the use of both ground-based and satellite-based cloud/atmospheric monitoring over high-resolution timescales for extended periods of time is required. In addition, information 
regarding potentially important microphysical properties such as aerosols, cloud droplet size, and atmospheric electricity must also be considered. Through such monitoring efforts, in addition to both computational modelling (such as that of Zhou and Tinsley, 2010) and experimental efforts (such as that of Duplissy et al., 2010) we may hope to better understand the effects described here.

Acknowledgements. The authors would like to thank Professor Loïs Steenman-Clark (University of Reading), Sir Arnold Wolfendale (Durham University), Tom Goren (University of Jerusalem), Professor Daniel Rosenfeld (University of Jerusalem), Johannes Quaas and two anonymous referees for their helpful comments. The NCEP Reanalysis Project data is provided by the NOAA/OAR/ERSL PSD, Boulder, Colorado, USA from http://www.cdc.noaa.gov/. Mg II index data were obtained from NOAA's Space Weather Prediction Center; the ISCCP D1 data are available from the ISCCP web site at http://isccp.giss.nasa.gov/, maintained by the ISCCP research group at the NASA Goddard Institute for Space Studies. IMF and TSI datasets were obtained from National Geophysical Data Center (NGDC) Space Physics Interactive Data Resource (SPIDR), and NASA's OMNI project. ACRIM data, obtained from http://www.acrim.com/Data\%20Products.htm.

Edited by: J. Quaas

\section{References}

Bond, G., Kromer, B., Beer, J., Muscheler, R., Evans, M., Showers, W., Hoffmann, S., Lotti-Bond, R., Hajdas, I., and Bonani, G.: Persistent solar influence on North Atlantic climate during the Holocene, Science, 294, 2130-2136, 2001.

Campbell, G.: View angle dependence of cloudiness and the trend in ISCCP cloudiness. In 13th Conference on Satellite Meteorology and Oceanography. Norfolk, American Meteorological Society, Boston, P6.7, 2004.

Carslaw, K., Harrison, R., and Kirkby, J.: Cosmic rays, clouds and climate, Science, 298, 1732-1737, 2002.

Douglas, D. and Clader, B.: Climate sensitivity of the Earth to solar irradiance, Geophys. Res. Lett., 29, 33-1, 2002.

Duplissy, J., Enghoff, M. B., Aplin, K. L., Arnold, F., Aufmhoff, H., Avngaard, M., Baltensperger, U., Bondo, T., Bingham, R., Carslaw, K., Curtius, J., David, A., Fastrup, B., Gagn, S., Hahn, F., Harrison, R. G., Kellett, B., Kirkby, J., Kulmala, M., Laakso, L., Laaksonen, A., Lillestol, E., Lockwood, M., Mkel, J., Makhmutov, V., Marsh, N. D., Nieminen, T., Onnela, A., Pedersen, E., Pedersen, J. O. P., Polny, J., Reichl, U., Seinfeld, J. H., Sipil, M., Stozhkov, Y., Stratmann, F., Svensmark, H., Svensmark, J., Veenhof, R., Verheggen, B., Viisanen, Y., Wagner, P. E., Wehrle, G., Weingartner, E., Wex, H., Wilhelmsson, M., and Winkler, P. M.: Results from the CERN pilot CLOUD experiment, Atmos. Chem. Phys., 10, 1635-1647, doi:10.5194/acp-101635-2010, 2010.

Evan, A. T., Heidinger, A. K., and Vimont, D. J.: Arguments against a physical long-term trend in global ISCCP cloud amounts, Geophys. Res. Lett., 34, L04701, doi:10.1029/2006GL02808, 2007.

Haigh, J.: The impact of solar variability on climate, Science, 272, 981-984, 1996.
Harrison, R. and Ambaum, M.: Observed atmospheric electricity effect on clouds, Environ. Res. Lett., 4, 014003-014008, 2009.

Harrison, R. and Stephenson, D.: Empirical evidence for a non linear effect of galactic cosmic rays on clouds, Proc. Roy. Soc. AMath. Phy., 462, 1221-1233, 2006.

Kalnay, E., Kanamitsu, M., Kistler, R., Collins, W., Deaven, D., Gandin, L., Iredell, M., Saha, S., White, G., Woollen, J., Zhu, Y., Chelliah, M., Higgins, W., Janowiak, J., Mo, K., Ropelewski, C., Wang, J., Leetmaa, A., Reynolds, R., Jenne, R., and Joseph, D.: The NCEP/NCAR 40-Year Reanalysis Project, Bull. Am. Met. Soc., 77, 437-471, 1996.

Kniveton, D., Sciare, M., and Mihalopoulous, J.: Variability of atmospheric dimethylsulphide over the southern Indian Ocean due to changes in ultraviolet radiation, Global Biogeochem. Cy., 14, 7.1-7.6, 2003

Kristjánsson, J. E., Staple, A., Kristiansen, J., and Kaas, E.: A new look at possible connections between solar activity, clouds and climate, Geophys. Res. Lett., 29, 22.1-22.4, 2002.

Kristjánsson, J. E., Stjern, C. W., Stordal, F., Fjæraa, A. M., Myhre, G., and Jónasson, K.: Cosmic rays, cloud condensation nuclei and clouds - a reassessment using MODIS data, Atmos. Chem. Phys., 8, 7373-7387, doi:10.5194/acp-8-7373-2008, 2008.

Laken, B. and Kniveton, D.: Forbush decreases and Antarctic cloud anomalies in the upper troposphere, J. Atmos. Sol. Terr. Phys., doi:10.1016/j.jastp.2010.03.008, in press, 2010.

Laken, B., Wolfendale, A., and Kniveton, D.: Cosmic ray decreases and changes in the liquid water cloud fraction over the oceans, Geophys. Res. Lett., 36, doi:10.1029/2009GL040961, L23803, 2010.

Laut, P.: Solar activity and terrestrial climate: an analysis of some purported correlations, J. Atmos. Sol. Terr. Phys., 65, 801-802, 2003.

Marsh, N. and Svensmark, H.: Low cloud properties influenced by cosmic rays, Phys. Rev. Lett., 85, 5004-5007, 2000.

Mauas, P., Flamenco, E., and Buccino, A.: Solar forcing of the stream flow of a continental scale South American river, Phys. Rev. Lett., 101, 168501-4, 2008.

Meehl, G. A., Arblaster, J. M., Matthes, K., Sassi, F., and van Loon, H.,: Amplifying the Pacific climate system response to a small 11-year solar cycle forcing, Science, 352, 1114-1118, 2009.

Neff, U., Burns, S., Mangini, A., Mudelsee, M., Fleitmann, D., and Matter, A.: Strong coherence between solar variability and the monsoon in Oman between 9 and 6 kyr ago, Nature, 411, 290293, 2001.

Nicoll, K. and Harrison, R.: Experimental determination of layer cloud edge charging from cosmic ray ionisation, Geophys. Res. Lett., 37, L13802, doi:10.1029/2010GL043605, 2010.

Pallé, E.: Possible satellite perspective effects on the reported correlations between solar activity and clouds, Geophys. Res. Lett., 32, L03802, doi:10.1029/2004GL021167, 2005.

Raes, F., Janssens, A., and van Dingenen, R.: The role of ioninduced aerosol formation in the lower atmosphere, J. Aerosol. Sci., 17, 466-470, 1986.

Rosenfeld, D., Kaufman, Y. J., and Koren, I.: Switching cloud cover and dynamical regimes from open to closed Benard cells in response to the suppression of precipitation by aerosols, Atmos. Chem. Phys., 6, 2503-2511, doi:10.5194/acp-6-2503-2006, 2006.

Rossow, W., Walker, A., Beuscel, D., and Roiter, M.: ISCCP 
documentation of new cloud datasets, World Met. Org. Tech. Doc., 737, 115 pp., 1996.

Svensmark, H.: Cosmoclimatology: a new theory emerges, Astron. Geophys., 48, 1.18-1.24, 2007.

Svensmark, H. and Friis-Christensen, E.: Variation of cosmic ray flux and global cloud coverage - a missing link in solar-climate relationships, J. Atmos. Sol. Terr. Phys., 59, 1225-1232, 1997.

Sun, B. and Bradley, R.: Solar influences on cosmic rays and cloud formation: a reassessment, J. Geophys. Res., 107, 0148-0227, 2002.

Tinsley, B.: The global atmospheric electric circuit and its effects on cloud microphysics, Rep. Prog. Phys., 71, 066801-066832, 2008.

Tinsley, B. and Dean, G.: Apparent tropospheric response to MeV$\mathrm{GeV}$ particle flux variations: a connection via electrofreezing of supercooled water in high-level clouds, J. Geophys. Res., 96, 22.283-22.296, 1991.

Tinsley, B. and Yu, F.: Atmospheric ionization and clouds as links between solar activity and climate, in: Solar Variability and its Effects on Climate, edited by: Pap, J. and Fox, P., Geophysical Monograph, 141, AGU Press, Washington, DC, 321-339, 2004.

Tinsley, B., Rohrbaugh, R., and Hei, M.: Electroscavenging in clouds with broad droplet size distributions and weak electrification, Atmos. Res., 59(60), 115-136, 2000.

Tinsley, B. A., Zhou, L. M., and Plemmons, A.: Changes in scavenging of particles by droplets due to weak electrification in clouds, Atmos. Res., 79, 266-295, 2006.

Tinsley, B., Burns, G., and Zhou, L.: The role of the global electric circuit in solar and internal forcing of clouds and climate, Adv. Space Res., 40, 1126-1139, 2007.

Todd, M. and Kniveton, D.: Changes in cloud cover associated with Forbush decreases of galactic cosmic rays, J. Geophys. Res., 106, 32031-32041, 2001.
Tripathi, S. and Harrison, G.: Enhancement of contact nucleation by scavenging of charged aerosol particles, Atmos. Res., 62, 5770, 2002.

van der Wal, A.: The Unified Model. Unified Model Users Guide, Version 2, The Meteorological Office: Reading, 192 pp., 1998.

Veretenenko, S., Dergachev, V., and Dmitriyev, P.: Solar activity and cosmic ray variations as a factor of intensity of cyclonic processes at midlatitudes, Geomagn. Aeronomy., 47, 0016-7932, 2007.

Yu, F., Wang, Z., Luo, G., and Turco, R.: Ion-mediated nucleation as an important global source of tropospheric aerosols, Atmos. Chem. Phys., 8, 2537-2554, doi:10.5194/acp-8-2537-2008, 2008.

Webb, M. J., Senior, C. A., Bony, S., Morcrette, J.-J.: Combining ERBE and ISCCP data to assess clouds in the Hadley Centre, ECMWF and LMD atmospheric models, Clim Dyn, 17, 905922, 2001.

Willson, R. C. and Mordvinov, A. V.: Secular total solar irradiance trend during solar cycles 21 23, Geophys. Res. Lett., 30, 11991202, 2003.

Zhang, M., Lin, W., Klein, S., Bacmeister, J., Bony, S., Cederwall, R., Del Genio, A., Hack, J., Loeb, N., Lohmann, U., Minnis, P., Musat, I., Pincus, R., Stier, P., Suarez, M., Webb, M. , Wu, J., Xie, S., Yao, M., and Zhang, J.: Comparing clouds and their seasonal variation in 10 atmospheric general circulation models with satellite measurements, J. Geophys. Res., 110, D15, 2005.

Zhou, L. and Tinsley, B.: Global circuit model with clouds, J. Atmos. Sci., 67, 1143-1156, 2010. 